\title{
DES FIBRES OPTIQUES \\ pour les environnements radiatifs extrêmes
}

\author{
Adriana MORANA ${ }^{1}$, Aziz BOUKENTER ${ }^{1}$, Youcef OUERDANE ${ }^{1}$, Emmanuel MARIN ${ }^{1}$, Sylvain GIRARD $^{1 *}$, \\ Gilles MELIN ${ }^{2}$, Laurent LABLONDE ${ }^{2}$, Arnaud LAURENT ${ }^{2}$, Thierry ROBIN ${ }^{2}$, Benoît CADIER \\ Laboratoire Hubert Curien, Saint-Etienne, France \\ iXblue Photonics, Lannion, France \\ *sylvain.girard@univ-st-etienne.fr; ; benoit.cadier@ixblue.com
}

Durant la dernière décennie, les besoins en fibres optiques et en capteurs à fibres optiques aptes à opérer dans les environnements radiatifs les plus extrêmes ont connu une forte augmentation : pouvoir anticiper et/ou monitorer les conditions d'exploitation et leurs évolutions est devenu une absolue nécessité dans une multitude de domaines que sont l'espace, l'industrie nucléaire, le stockage des déchets radioactifs, ou encore la physique des hautes énergies et installations dévolues à l'étude de la fusion (Laser Mégajoule, ITER).

\begin{abstract}
I a technologie des fibres optiques à base de silice et des capteurs basés sur ces composants offre de nombreux avantages pour une intégration dans des environnements sévères. En effet, les fibres optiques présentent de faibles poids et volumes, une excellente résistance aux perturbations électromagnétiques, et peuvent grâce à des revêtements métalliques résister à des températures supérieures à $300^{\circ} \mathrm{C}$. Les applications visées sont nombreuses telles que le transfert de données, le contrôle-commande, la réalisation de sources optiques ou amplificateurs, de diagnostics plasma ou laser, et bien sûr de capteurs ponctuels ou répartis de température, déformation, pression, vibration ou niveaux de liquide. Certains de ces environnements sont associés à des contraintes radiatives qu'il est possible de définir grâce à trois paramètres : la nature de l'irradiation (photons X \& gamma, ions, neutrons ou protons), la dose (ou fluence) déposée qui s'exprime en Gray $(1 \mathrm{~Gy}=1 \mathrm{~J} / \mathrm{kg})$ et le débit de dose (ou flux, Gy/s) qui caractérise la vitesse de dépôt de cette énergie. La figure 1 illustre les différents environnements radiatifs pour lesquels des études sont menées par
\end{abstract}

le laboratoire commun LabH6, laboratoire qui regroupe le laboratoire Hubert Curien (UJM, CNRS, IOGS) et la société iXblue, en vue d'établir la vulnérabilité des solutions à base de fibres optiques ou en vue d'améliorer leur tolérance (on parle alors d'études de durcissement) avant leur implémentation sur site.

\section{Les effets des radiations suri les fibres optiques Les effets macroscopiques}

Lors de l'exposition d'une fibre optique aux radiations, trois principaux effets macroscopiques sont observés. Le premier, limitant le plus souvent les applications visées, est l'atténuation induite par irradiation (ou RIA). Sous irradiation, l'atténuation linéique de la fibre, très faible avant irradiation $(0,2 \mathrm{~dB} / \mathrm{km}$ à $1550 \mathrm{~nm}$ ) augmente fortement avec des cinétiques qui different d'une fibre optique à l'autre et aussi en fonction du profil d'emploi de la fibre : longueur d'onde du signal, puissance injectée... Lorsque l'irradiation cesse, une partie des excès de pertes optiques sont recouvrées, là aussi avec des cinétiques très différentes selon le type de fibre optique.

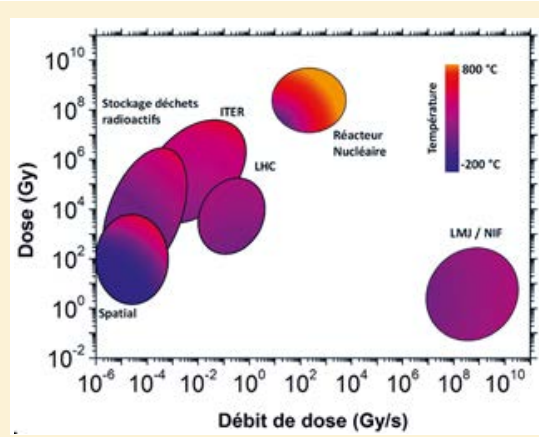

Figure 1. Les différents environnements radiatifs d'intérêt pour les fibres optiques et capteurs à fibres optiques.

De nombreux travaux ont été menés depuis les années 1970 afin d'identifier les paramètres intrinsèques ou extrinsèques aux fibres optiques qui affectent les niveaux et cinétiques de croissance et déclin de la RIA [1]. Parmi tous ces paramètres, la composition du cœur et de la gaine optique de la fibre joue un rôle primordial, tout comme la dose déposée, le débit de dose et la température de l'irradiation. Sur la figure 2 sont comparées les cinétiques de croissance de la RIA à $1550 \mathrm{~nm}$ de fibres différemment dopées en cœur lors d'une exposition continue à des photons $\mathrm{X}$ de $40 \mathrm{keV}$. Ainsi, il est visible que les fibres contenant $\mathrm{du}$ 


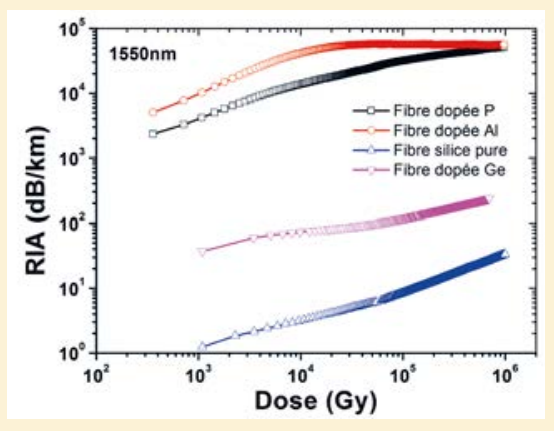

Figure 2. RIA induite à $1550 \mathrm{~nm}$ en fonction de la dose pour différentes compositions de fibres optiques [2].

phosphore ou de l'aluminium, telles que les fibres amplificatrices erbium (Er) ou erbium-ytterbium (ErYb), sont très sensibles aux radiations, tandis que celles à cour de silice pure présentent la meilleure tenue face à ce type d'environnement. Les radiations peuvent également causer deux autres phénomènes: le premier concerne l'émission de lumière induite par irradiation, le second celui de compaction induite par irradiation amenant, entre autres à un changement de l'indice de réfraction, particulièrement préjudiciable pour les capteurs à fibres optiques.

\section{Les origines microscopiques}

Pour pouvoir modifier la réponse radiative d'une fibre optique, il est indispensable de comprendre et de contrôler les mécanismes physiques à l'origine de son atténuation induite par irradiation ou de sa radioluminescence. À l'échelle microscopique, celles-ci sont causées par la génération par déplacements atomiques ou ionisation de défauts ponctuels dans la silice $\left(\mathrm{SiO}_{2}\right)$ pure ou dopée constituant le cœur et la gaine optique des fibres optiques [2]. Ces structures de défauts, différentes selon les dopants ou impuretés présents dans la matrice vitreuse, sont associées à des bandes d'absorption optique, pour certaines à des bandes de luminescence, expliquant les phénomènes de RIA (radiation induced attenuation), RIE (radiation induced emission) et pour partie celui de compaction. La majorité des bandes d'absorption sont localisées dans l'ultraviolet et le visible, ce qui explique que les pertes mesurées dans ces domaines spectraux sont généralement plus élevées que celles relevées dans l'infrarouge. Afin d'identifier les défauts expliquant la vulnérabilité d'une solution fibrée et d'imaginer des techniques de contrôle de leur efficacité de génération, il est nécessaire de combiner des études théoriques, multi-échelles et multi-physiques, à des mesures spectroscopiques sur des échantillons spécifiquement dédiés à ces études. Ces travaux sont menés en collaboration entre le LabH6 et le CEA DAM de Bruyères-le-Châtel [2] sur des fibres canoniques réalisées spécifiquement pour ces études par iXblue.

\section{Les avancées marquantes}

La compréhension accrue des mécanismes à l'origine de la réponse radiative des fibres optiques a permis la définition, la réalisation et l'intégration de solutions fibrées dans différents domaines du nucléaire. Certaines des avancées récentes sont décrites ci-après, avec des applications concrètes dans le domaine des communications et télécommunications spatiales ou encore dans le domaine de la dosimétrie.

\section{Les applications spatiales}

Qu'il s'agisse du déploiement de constellations de satellites, ou de sources optiques de type Lidar pour les missions scientifiques spatiales, le développement de fibres optiques spéciales résistantes aux radiations est nécessaire à la réalisation des futurs systèmes optiques de communication en espace libre. Les sources et amplificateurs optiques à base de fibres dopées erbium ou erbiumytterbium en sont un élément clef [3]. La réponse des composants non-durcis est fortement affectée par les radiations, avec une très forte dégradation de leur gain sous irradiation (cf. figure 3). Cette dégradation s'explique par la matrice vitreuse du cœur de ces fibres, généralement phosphosilicate ou aluminosilicate. Des techniques de durcissement ont été identifiées par le LabH6, en collaboration avec le CNES, telles que l'addition de cerium ou le chargement en hydrogène de structures spécifiques de fibres optiques. Ces solutions permettent de réduire drastiquement la RIA aux longueurs d'onde de pompe et du signal et de conserver ainsi un gain stable durant toute la mission. Des travaux combinant simulations et expérimentations sont en cours pour travailler à un durcissement supplémentaire au niveau système des amplificateurs optiques de très forte puissance (>10 W à $1550 \mathrm{~nm}$ ) où des effets thermiques et radiatifs se combinent pour dicter la réponse radiative de ces systèmes [3].

\section{Des fibres optiques durcies dans l'infrarouge}

De très nombreuses applications (sources et amplificateurs, lidar, gyroscope à fibres optiques) nécessitent l'usage de fibres optiques passives, monomodes ou multimodes, fonctionnant dans les fenêtres des télécommunications sous irradiation gamma à des doses supérieures à 100 kGy. Elles peuvent servir pour le transfert de données mais aussi en tant qu'éléments sensibles de capteurs à fibres optiques : capteurs distribués (basés sur la rétrodiffusion Raman, Rayleigh ou Brillouin) de température ou de déformation pour le stockage des déchets radioactifs, de niveau d'eau pour les piscines nucléaires... [4]. Afin de minimiser les niveaux de RIA, il convient à la fois de travailler sur la composition de la fibre optique, généralement à cœur de silice pure ou dopée fluor, mais aussi sur ses paramètres de fabrication : conditions de dépôt de la préforme et conditions d'étirage de façon à repousser les seuils d'activation de création de défauts structurels au cœur de la matrice vitreuse et à minimiser ainsi

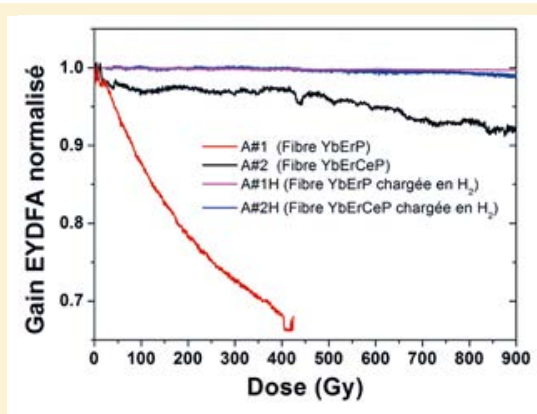

Figure 3. Performances d'amplificateurs optiques $\mathrm{Er} / \mathrm{Yb}$ basés sur une fibre standard (A\#1), codopée Ce (A\#2) et des versions hydrogénées de ces fibres $(\mathrm{A \# 1 \textrm {H }}$ et $1 \# 2 \mathrm{H})$. 
les prises d'atténuation [4]. Aujourd'hui, les meilleures fibres optiques présentent des pertes à $1550 \mathrm{~nm}$ de l'ordre de 10 à $30 \mathrm{~dB} / \mathrm{km}$ sous irradiation à $1 \mathrm{MGy}$ soit près d'un facteur 1000 par rapport à une fibre non durcie. De plus, à ces niveaux de dose, il convient de s'assurer que la tenue mécanique de la fibre optique n'évolue pas sous irradiation, en particulier lorsque la contrainte radiative est couplée à de fortes températures, l'utilisation de revêtement polyimide est alors à privilégier [4].

\section{Des fibres optiques radiosensibles pour la dosimétrie}

Si la majorité des études sont dédiées à l'amélioration de la réponse des fibres optiques face aux radiations, il est également établi que certaines fibres optiques radiosensibles, en particulier celles dopées au phosphore permettent de mesurer la dose ionisante de radiations, de manière ponctuelle ou répartie via la mesure de RIA ou de RIE. Le LabH6 collabore sur cette thématique avec le CERN qui travaille actuellement à définir et qualifier une solution de dosimétrie répartie, permettant de fournir une cartographie 1D de la dose ionisante au sein des environnements très complexes associés à leurs accélérateurs [5]. En effet, en combinant une fibre radiosensible phosphosilicate à un réflectomètre optique (optical time domain reflectometer, OTDR ou optical frequency domain reflectometer, OFDR) il devient possible de mesurer l'évolution locale des pertes linéiques en raison de la prise d'atténuation locale; la dose peut alors être mesurée le long d'une fibre avec une résolution sub-métrique. Pour concevoir un dosimètre performant, il convient que ces fibres présentent d'autres caractéristiques particulières. En effet, la RIA ne doit, par exemple, pas dépendre du débit de dose, de la température (dans les gammes visées par l'application, jusqu'à $50{ }^{\circ} \mathrm{C}$ pour les besoins CERN) pour assurer une mesure fiable [5]. Au regard de tous ces paramètres, les fibres phosphosilicates sont aujourd'hui les plus performantes mais des travaux restent en cours pour étendre les gammes de

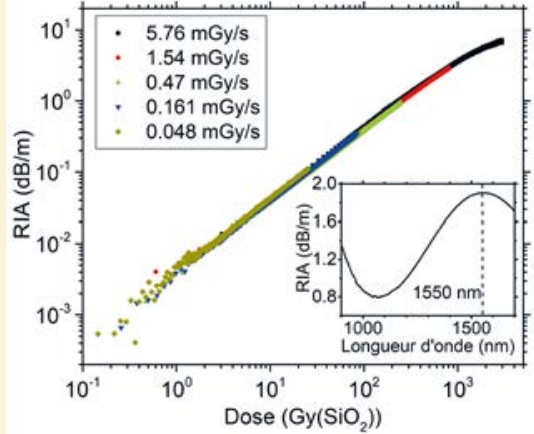

Figure 4. Évolution de l'atténuation induite par irradiation (RIA) à $1550 \mathrm{~nm}$ en fonction de la dose, du débit de dose pour une fibre optique dopée P [5].

dose accessibles (faibles et fortes doses) ou identifier des solutions permettant de discriminer différents types de radiations (gamma, neutrons, protons...).

\section{Conclusion.}

Les fibres optiques sont de plus en plus envisagées pour instrumenter les environnements les plus sévères en termes de contraintes radiatives. Aujourd'hui, grâce à une compréhension de plus en plus accrue des mécanismes à l'origine de leur dégradation sous irradiation, il devient possible de les fonctionnaliser soit pour renforcer leur tolérance face aux radiations, en minimisant en particulier leur atténuation induite par irradiation, soit pour l'exacerber afin de concevoir des dosimètres répartis innovants. Ces avancées ouvrent de très prometteuses perspectives en vue de l'instrumentation des environnements les plus sévères, en particulier dans les domaines du spatial, de la physique des hautes énergies, des installations de fusion et fission mais aussi potentiellement pour la médecine.

\section{POUR EN SAVOIR PLUS}

[1] S. Girard et al., IEEE Transactions on Nuclear Science 60, 2015 (2013)

[2] S. Girard et al., Reviews in Physics 4, 100032 (2019)

[3] S. Girard et al., Journal of Optics 20, 093001 (2018)

[4] G. Mélin et al., IEEE Transactions on Nuclear Science 66, 1657 (2019)

[5] D. Di Francesca et al., IEEE/OSA Journal of Lightwave Technology, in press, https:// doi.org/10.1109/JLT.2019.2915510

\section{Cellule de Pockels BBO}

- Taux de répétition $>\mathbf{2} \mathbf{~ M H z}$

- Transmission $>98 \%$

- Contraste VCR > 1:1000

- Puissance moyenne pour lasers ultra-rapides $>100 \mathrm{~W}$

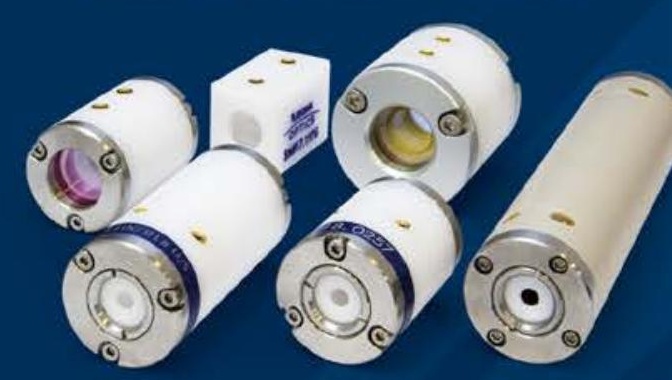

APPLICATIONS

- Selection d'impulsions ou train d'impulsions

- Injection/extraction de pulse dans amplificateur régénératif

- Q-switching

- Modulation de laser CW

\section{Driver de cellule de Pockels, avec alimentation dédiée}

- Pour des opérations au-delà du Mhz

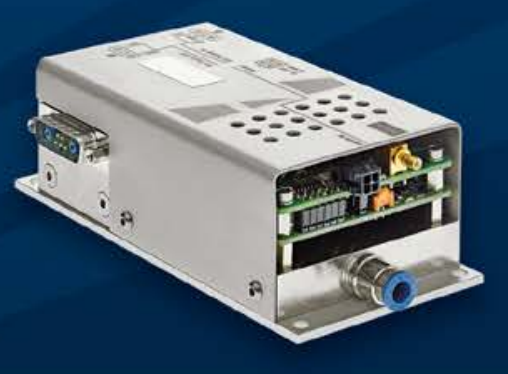

www.eksmaoptics.com

Représenté en France par:

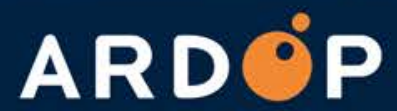

INDUSTRIE 